\title{
Optical clocks based on stimulated emission radiation
}

\author{
WANG YiQiu \\ School of Electronics Engineering and Computer Science, Peking University, Beijing 100871, China
}

Atomic clocks operating at microwave region, are divided into two categories according to the mode of operation, namely the passive mode, in which the standard frequency of a clock signal is locked on to a very narrow atomic spectral line such as in cesium beam and rubidium gas cell clocks, and the active mode, where the atomic ensemble itself can produce a high stable and accurate standard frequency signal such as hydrogen maser. The hydrogen maser has been the most stable atomic clock for nearly half a century, and has been applied in a variety of scientific research fields ${ }^{[1]}$.

The optical clocks, operating at frequency domain by about five orders of magnitudes higher than that of the microwave clocks, have been expected to surpass the performance of microwave clocks both in stability and uncertainty. With the development and maturity of femtosecond optical frequency comb ${ }^{[2,3]}$, very recently, the ion optical clock ${ }^{[4]}$ and neutral Sr optical clock ${ }^{[5]}$ have demonstrated better stability and uncertainty than any microwave clocks. However, all the optical clocks realized up to date are based on the "passive mode" of operation, this means that the laser frequency is locked to the very narrow atomic spectral line, but not the lasing light (the stimulated emission radiation) frequency itself which is very stable and can be used as a frequency standard — the active mode.

Recently, Chen has proposed the concept of active

1 Riehle F. Frequency standards: Basics and applications. Weinheim: Wiley-VCH Verlag GmbH \& Co KGaA, 2004

2 Hall J L. Nobel lecture: Defining and measuring optical frequencies. Rev Mod Phys, 2006, 78: 1279-1295

3 Hansch T W. Nobel lecture: Passion for precision. Rev Mod Phys, 2006, 78: 1297-1309

4 Rosenband T, Hume D B, Schmidt P O, et al. Frequency ratio of $\mathrm{Al}^{+}$ and $\mathrm{Hg}^{+}$single-ion optical clocks; metrology at the 17th decimal place. Science, 2008, 319(5871): 1808-1812 optical clock (see ref. [6] in this issue), which is an optical clock based on the stimulated emission radiation. This is the first extension of the principle of hydrogen maser from microwave domain to optical domain. There are twofold advantages of this active optical clock. On one hand, the linewidth of the output laser from active optical clock determined by the modified SchawlowTownes formula ${ }^{[7]}$, is much narrower than the linewidth due to transit time, which is the ultimate limit of absorption laser spectroscopy. On the other hand, utilizing bad cavity in active optical clock, the shift and noise of the centre frequency of active optical clock caused by cavity length change is reduced to a cavity pulling effect. Currently, the cavity length noise is the ultimate limit of existing lasers with coherence time longer than one second, and thus the laser linewidth is at $\mathrm{mHz}$ level.

Active optical clocks provide several new possibilities of applications: (i) more stable optical clock than any current atomic clocks; (ii) sub-natural linewidth laser spectroscopy; (iii) long coherence time laser with linewidth at $\mathrm{mHz}$ level; (iv) Ramsey laser combining stimulated emission process and Ramsey separated oscillatory fields method.

Therefore, the prospect of active optical clock is attractive for the research at the field of optical clock, laser spectroscopy and metrology.

5 Ludlow A D, Zelevinsky T, Campbell G K, et al. Sr lattice clock at $1 \times 10^{-16}$ fractional uncertainty by remote optical evaluation with a $\mathrm{Ca}$ clock. Science, 2008, 319: 1805-1808

6 Chen J B. Active optical clock. Chin Sci Bull, 2009, 54(3): 348-352

7 Yu D S, Chen J. Laser theory with finite atom-field interacting time. Phys Rev A, 2008, 78: 013846

doi: 10.1007/s11434-009-0064-Z email: wangyq@pku.edu.cn 